\title{
HIPERTENSÃO ARTERIAL COMO FATOR DE RISCO PARA DOENÇAS CARDIOVASCULARES
}

\author{
ARTERIAL HYPERTENSION AS A RISK FACTOR FOR CARDIOVASCULAR DISEASES
}

Marcus Vinicius Simões ${ }^{1}$, André Schmidt ${ }^{2}$

Docente $^{1}$, Médico Assistente ${ }^{2}$ da Seção de Cardiologia do Departamento de Clínica Médica da Faculdade de Medicina de Ribeirão Preto da Universidade de São Paulo

CoRRESPONDÊnCIA: Marcus Vinicius Simões, Hospital das Clínicas da Faculdade de Medicina de Ribeirão Preto da Universidade de São Paulo - Campus Universitário - Seção de Cardiologia - CEP: 14048-900 - Ribeirão Preto - SP

SIMÕES MV \& SCHMIDT A. Hipertensão arterial como fator de risco para doenças cardiovasculares. Medicina, Ribeirão Preto, 29: 214-219, abr./set. 1996.

RESUMO: Evidências, recentemente, extraídas de amplos estudos epidemiológicos confirmaram a participação da hipertensão arterial sistêmica (HAS) como fator de risco maior para desenvolvimento de complicações cardiovasculares. Nesta breve revisão do assunto, as características clínicas da HAS que interferem, diretamente, na determinação do risco de complicações cardiovasculares degenerativas de natureza aterosclerótica são apresentadas, dando-se destaque à avaliação do risco individual. Aspectos recentes da participação da hipertrofia ventricular esquerda, como preditor de risco de morte súbita arrítmica, são também objetivamente revistos.

UNITERMOS: Hipertensão. Fator de Risco. Aterosclerose.

\section{INTRODUÇÃO}

É, relativamente, bem conhecido na prática clínica que regime pressórico persistente elevado ao longo do tempo, mesmo naqueles indivíduos assintomáticos, resulta em importante morbidade e mortalidade decorrentes de doenças cardiovasculares. Da doença hipertensiva não tratada resultam duas formas de acometimento vascular degenerativo. Primeiramente, aquelas diretamente associadas à hipertensão por si própria, podendo ser encaradas como complicações da história natural da doença hipertensiva. Estas complicações vasculares hipertensivas podem apresentar evolução fatal conseqüente à insuficiência renal, insuficiência cardíaca e acidente vascular cerebral hemorrágico ${ }^{1}$. Em outro plano, situam-se as alterações degenerativas do sistema vascular de natureza aterosclerótica que são agravadas ou aceleradas pela hipertensão arterial, particularmente, a doença arterial coronariana, pela sua elevada incidência (Tabela I). Neste contexto, a doença hipertensiva constitui importante fator de risco para o desenvolvimento da doença aterosclerótica.

Levantamentos epidemiológicos, obtidos através do seguimento longitudinal de grandes populações ao longo de várias décadas, foram claramente conclusivos a respeito da participação da Hipertensão Arterial Sistêmica (HAS) na determinação de morbi-mortalidade cardiovascular. Nos estudos oriundos da população de Framingham, seqüelas cardiovasculares ateroscleróticas, incluindo acidente vascular cerebral átero-trombótico, doença cardíaca coronariana e doença arterial periférica, ocorreram com frequiência global 2 a 3 vezes maior em hipertensos, quando comparados com normotensos da mesma idade ${ }^{2}$. 
Tabela I. Complicações cardiovasculares associadas à hipertensão

\section{A. Hipertensivas}

- Hipertensão maligna

- Acidente vascular cerebral hemorrágico

- Insuficiência cardíaca congestiva

- Nefroesclerose

- Dissecção aórtica.

\section{B. Ateroscleróticas}

- Doença arterial coronariana

- Morte súbita

- Acidente vascular cerebral isquêmico (átero-trombótico)

- Doença obstrutiva arterial periférica.

Conforme estes dados demonstram (Figura 1), o risco relativo induzido pela presença de HAS é maior quando se considera a ocorrência de complicações vasculares cerebrais, do que quando se contempla a ocorrência de doença obstrutiva arterial coronária. Contudo, levando-se em conta a alta incidência desta última, particularmente o infarto miocárdico, esta complicação aterosclerótica, associada à HAS, constitui-se na de maior importância no âmbito da saúde pública.
Um aspecto clínico pouco compreendido da associação entre HAS e doença arterial coronariana é o aumento da ocorrência de infarto miocárdico assintomático ${ }^{3,4}$. Em indivíduos hipertensos do gênero masculino e feminino, $35 \%$ e $45 \%$, respectivamente, dos infartos não são reconhecidos clinicamente. Considerando-se que infartos assintomáticos acarretam um prognóstico, a longo prazo, tão sombrio quanto o infarto clinicamente expresso, a monitorização períodica do ECG em pacientes hipertensos, a fim de detectar IAM silentes, parece justificada.

\section{INTER-RELAÇÃO ENTRE HAS E DOENÇA ATE- ROSCLERÓTICA}

Os mecanismos pelos quais a HAS contribui para o desenvolvimento da doença vascular é complexo e, ainda, pouco entendido. Um aspecto fundamental da patogênese da aterosclerose que deve ser salientado é que, habitualmente, existem cofatores aterogênicos interagindo, com efeito multiplicativo ao da eventual hipertensão arterial como a dislipidemia, intolerância à glicose, tabagismo e obesidade. Contudo, parece claro que a HAS desempenha papel central nesse processo, uma vez que o processo aterosclerótico significativo, raramente, ocorre em segmentos do sistema circulatório com baixo regime pressórico, tais como artérias pulmonares ou veias, a despeito da sua exposição aos mesmos fatores circulantes aterogênicos.

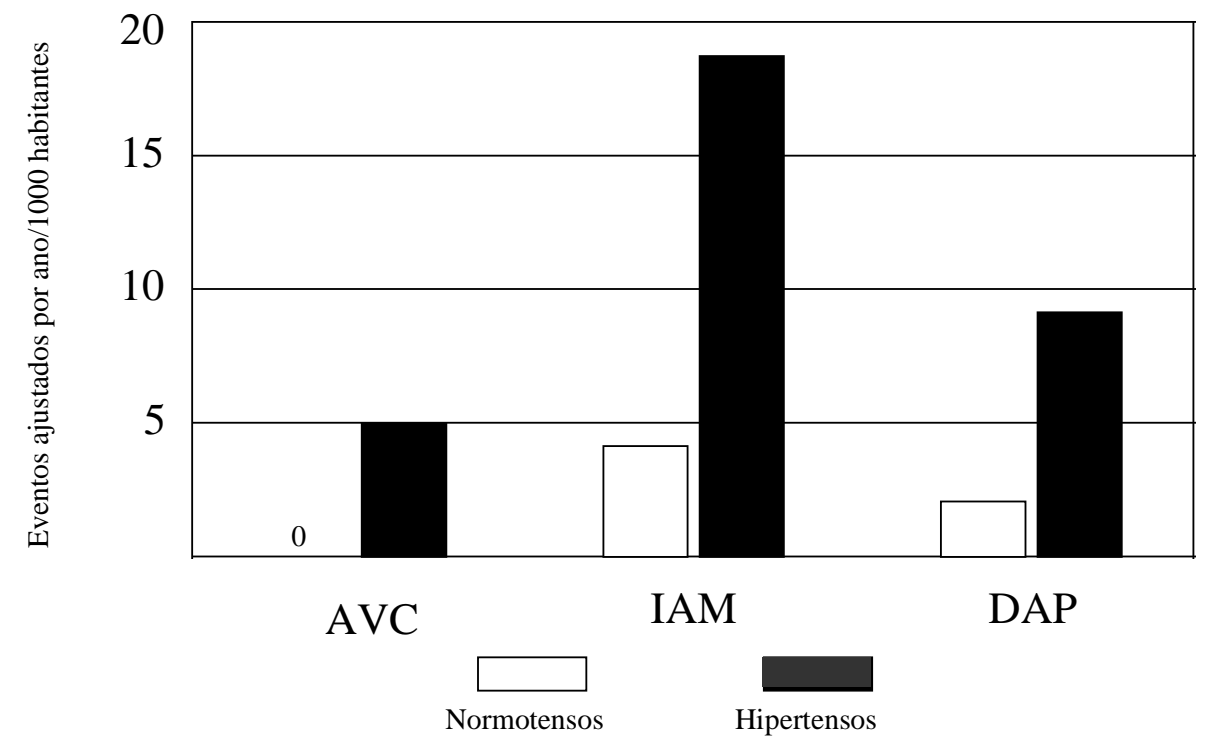

Figura 1. Incidência de eventos cardiovasculares ateroscleróticos, conforme o estado pressórico em indivíduos de 35 a 64 anos, de ambos os gêneros, resultado de 30 anos de seguimento - Framingham Study. AVC = acidente vascular cerebral isquêmico; IAM = infarto agudo do miocárdio; DAP = doença arterial periférica. 
Provavelmente, 3 fatores contribuem majoritariamente para este processo:

1. Pulsatilidade arterial. A degeneração das artérias é acelerada conseqüente às mudanças nas características mecânicas da onda de pulso arterial, maior pressão de pico, maior amplitude de oscilação, maior variabilidade e maior taxa de variação. Esses fatores de estresse parietal agem na produção de adelgaçamento das paredes, fratura das fibras elásticas e fibrosas, dilatação e, por fim, na formação de aneurismas com sua posterior ruptura.

2. Disfunção endotelial. As células endoteliais constituem um sistema secretor local de importantes moduladores do tônus vascular, tendo-se identificado tanto fatores relaxadores como constrictores, derivados destas células. Alterações da função endotelial podem participar, primariamente, na gênese da hipertensão arterial (insuficiência de substâncias vasodilatadoras endoteliais) ou participar do espectro de dano vascular, em conseqüência desta última. Particularmente, tem-se demonstrado que a disfunção endotelial pode participar nas etapas iniciais da deposição de lípides na íntima vascular que levam à formação de placas de ateroma. Paralelamente, alterações dos níveis séricos das lipoproteínas são capazes de induzir disfunção do endotélio. Essa inter-relação reforça o laço existente entre HAS e doença aterosclerótica.

3. Hipertrofia das células musculares lisas vasculares. Em resposta ao estresse parietal aumentado, ocorre hiperplasia das células musculares que contribui tanto para o processo de formação da placa de ateroma, como para perpetuação e progressão do distúrbio hipertensivo.

\section{CARACTERÍSTICAS CLÍNICAS DA PRESSÃO ARTERIAL ELEVADA E SUA RELAÇÃO COM RISCO DE COMPLICAÇÕES CARDIOVASCU- LARES}

\subsection{Elevação da pressão sistólica versus pressão diastólica}

Há crença de que a elevação do componente diastólico da pressão arterial, a despeito do componente sistólico aumentado, seja um determinante maior da sua gravidade clínica, implicando em elevação do risco de complicações. Os dados extraídos dos estudos de Framingham e do MRFIT (Multiple Risc Factor Intervention Trial) não confirmaram tais expectativas. Em todas as faixas etárias não se documentou impacto maior da pressão arterial diastólica do que a do componente sistólico na determinação de risco para desenvolvimento de doença cardiovascular ${ }^{5}$. Do mesmo modo, naqueles indivíduos com elevação dos valores sistólicos, a consideração dos valores diastólicos não trouxe informação adicional sobre risco cardiovascular. Em contrapartida, em indivíduos com hipertensão diastólica, a participação da elevação do componente sistólico era altamente informativa sobre o risco de eventos (Figura 2).

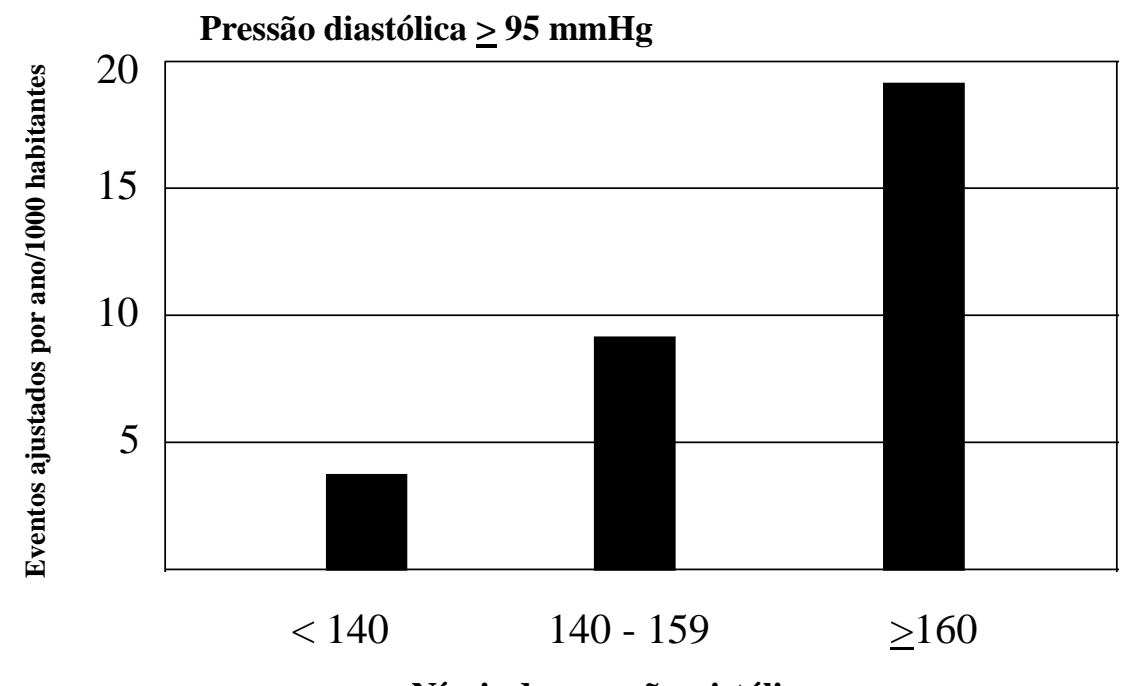

Níveis de pressão sistólica

Figura 2. Risco de acidente vascular cerebral, conforme os valores pressóricos sistólicos em indivíduos masculinos de 35 a 84 anos de idade, portadores de pressão diastólica $>95 \mathrm{mmHg}$. Resultados do Framingham Study, 26 anos de seguimento. 


\subsection{Hipertensão sistólica isolada.}

Com o progredir da idade, os níveis pressóricos diastólicos perdem progressivamente o seu impacto sobre a mortalidade cardiovascular. O processo de envelhecimento se acompanha de elevação progressiva da pressão sistólica secundária ao enrijeci- mento da parede das artérias. Antes de constituir-se em um processo benigno vinculado ao envelhecimento natural, o desenvolvimento da hipertensão arterial sistólica isolada em todas as idades, principalmente nos faixas etárias avançadas, é um determinante isolado de risco de eventos cardiovasculares (Figura 3$)^{6}$.

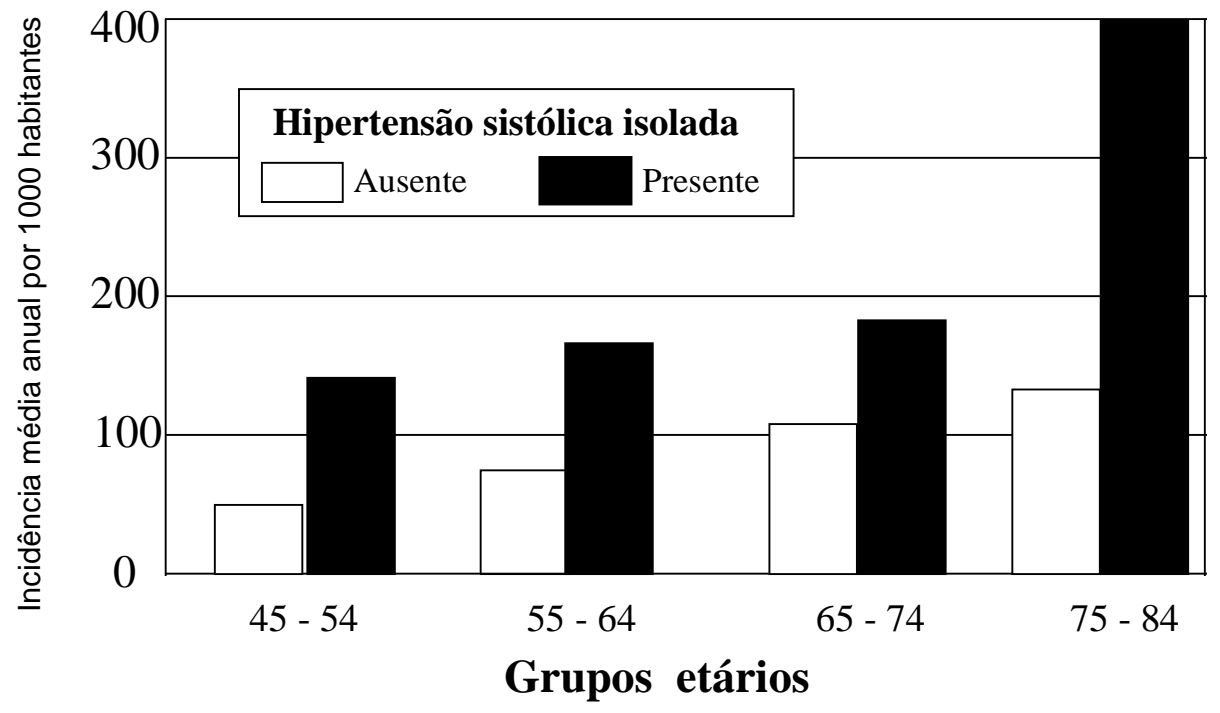

Figura 3. Risco de ocorrência de infarto agudo miocárdico em indivíduos masculinos, conforme a idade e presença de hipertensão arterial sistólica (pressão sistólica > $160 \mathrm{mmHg}$ e pressão diastólica < $95 \mathrm{mmHg}$ ). Framingham Study, 24 anos de seguimento.

\section{AVALIAÇÃO INDIVIDUAL DO RISCO DE COM- PLICAÇÕES CARDIOVASCULARES}

Parece ser claro haver uma razoável variabilidade individual quanto à propensão para desenvolvimento de doença vascular mais agressiva em portadores de níveis semelhantes de hipertensão arterial. Essa constatação sugere a necessidade de uma abordagem individual de aferição de risco de complicações cardiovasculares. Numa abordagem clínica prática de avaliação do risco individual de um determinado paciente portador de hipertensão leve, deve-se levar em conta além da coexistência de outros determinantes de risco cardiovascular (gênero masculino, idade avançada, elevação dos lípides séricos, tabagismo e intolerância à glicose), a evidência de acometimentos de órgãos-alvo: vasos retinianos, coração, rins e circulação cerebral. Nesse contexto, é de particular interesse a detecção de acometimento cardíaco, principalmente da presença de hipertrofia ventricular esquerda.

\subsection{Hipertrofia ventricular esquerda na Hiper- tensão Arterial Sistêmica, como fator inde- pendente de risco}

A hipertrofia ventricular esquerda (HVE) que ocorre na Hipertensão Arterial Sistêmica, consiste na via final do processo adaptativo funcional em resposta à elevação da resistência arterial periférica. Ao ser submetido a uma sobrecarga pressórica, o ventrículo tende a dilatar como resposta inicial, visando otimização do processo contrátil, através do mecanismo 
de Frank-Starling. Tal processo acarreta uma elevação no estresse da parede ventricular, segundo a Lei de Laplace. Para reduzir o diâmetro da cavidade, ocorre a hipertrofia da parede ventricular com o objetivo de reestabelecer o equilíbrio funcional ${ }^{7}$. O grau de hipertrofia correlaciona-se com os níveis pressóricos, mas também é influenciado por fatores genéticos, ambientais, bioquímicos e humorais. Conseqüências funcionais desta resposta fisiológica são variadas. $\mathrm{O}$ aumento da massa ventricular determina uma maior demanda de oxigênio e nutrientes para a célula, cujo metabolismo foi elevado em função do aumento no número de unidades contráteis. Para que seja reestabelecido o equilíbrio entre oferta e demanda de oxigênio, utiliza-se a reserva coronariana que, ao esgotarse, desencadeia o aparecimento de isquemia miocárdica e, posteriormente, necrose celular, substrato adequado para uma plêiade de alterações com repercussões prognósticas na HAS, propiciando o aparecimento de arritmia ventricular vinculada à ocorrência de morte súbita. Estudos provenientes de Framingham determinaram a importância da hipertrofia ventricular como fator de risco através de achados eletrocardiográficos, que foram confirmados, mais recentemente, por estudos ecocardiográficos, método mais sensível e específico que o, previamente, utilizado ${ }^{8}$.

\subsection{Relação entre HVE e arritmias ventriculares}

A patogênese da arrritmias ventriculares em pacientes com HVE é multicausal, com vários mecanismos possíveis descritos ${ }^{7,8}$. A existência de áreas de fibrose, um dos substratos presentes, favorece a ocorrência de fenômenos de reentrada, responsável por arritmias em várias situações fisiopatológicas. Outros mecanismos eletrofisiológicos foram descritos, como por exemplo, prolongamento do potencial de ação, dispersão de refratariedade e atividade deflagrada. A presença de isquemia miocárdica é mecanismo desencadeador potencial, por determinar alterações no limiar de excitabilidade celular e o aparecimento de impulsos ectópicos. A distensão do miócito é outro mecanismo possível, pois aumenta a suscetibilidade à despolarização espontânea.

A literatura tem demonstrado a correlação positiva entre a presença de HVE e a ocorrência de arritmia ventricular. Messerli e colaboradores, em $1984^{9}$, relataram a relação entre a presença de HVE em hipertensos e a maior prevalência de arritmias, quando comparados com hipertensos sem HVE e in- divíduos normotensos ${ }^{10}$. Tais resultados foram corroborados por McLenachan e colaboradores em 1987, em grupo de hipertensos que tiveram coronariopatia excluída por avaliação angiográfica, afastando potencial substrato fisiopatológico que poderia interferir na análise ${ }^{10}$. Trabalho recente, demonstrou a existência de um ritmo circadiano na ocorrência de arritmia ventricular, com um pico matutino e um vespertino ${ }^{11}$. Convém ressaltar que há uma grande variabilidade na ocorrência de arritmia ventricular, quando avaliada pela eletrocardiografia dinâmica. Outro relato interessante foi feito por James e Jones ${ }^{12}$, que encontraram arritmia em pacientes hipertensos com HVE, apenas quando havia hipocalemia concomitante. A ocorrência de distúrbios hidro-eletrolíticos é uma causa de arritmia conhecida e, potencialmente, muito relevante na HAS, pelo uso freqüente de diuréticos expoliadores de potássio.

\subsection{Hipertensão como fator de risco para morte súbita}

A ocorrência de arritmias ventriculares em um indivíduo com hipertrofia ventricular tem sido demonstrada como fator responsável por pior prognóstico em caso de infarto agudo do miocárdio, com aumento da área infartada e maior número de mortes por taquiarritmias. Taquiarritmias precedem a morte súbita em pacientes com hipertensão e hipertrofia ventricular esquerda ${ }^{13}$. A coexistência de doença arterial coronariana é um fator de risco adicional para elevar a morbi-mortalidade. Observou-se, ainda, uma coincidência entre a ocorrência de taquiarritmias ventriculares e morte súbita, o aparecimento de isquemia miocárdica silenciosa durante a eletrocardiografia dinâmica e elevações da pressão arterial, na forma de ritmos circadianos coincidentes. Se há uma inter-relação entre tais fenômenos, ainda, é matéria de controvérsia. Contudo, é evidente que há maior número de pacientes com morte súbita que apresentam hipertrofia ventricular.

Finalmente, o impacto da redução da hipertrofia ventricular sobre a mortalidade, decorrente de morte súbita não foi ainda comprovada ${ }^{14}$, sendo necessária a realização de estudos para determinar a necessidade de terapêutica específica antiarrítmica nos hipertensos com HVE, com e sem doença arterial coronariana comcomitante, e qual seria o medicamento ideal, em virtude dos múltiplos mecanismos responsáveis pela gênese de arritmias na HAS. 
SIMÕES MV \& SCHMIDT A. Arterial hypertension as a risk factor for cardiovascular diseases. Medicina, Ribeirão Preto, 29: 214-219, apr./sept. 1996.

ABSTRACT: Recent epidemiologic studies confirmed the role of arterial hypertension as a major risk factor for cardiovascular disease. A brief review of the hypertension clinical hallmarks regarding its repercussion on risk determination of atherosclerotic disease is presented. Concepts about left ventricular hypertrophy as a predictor of sudden death by arrythmics mechanisms are objectively updated.

UNITERMS: Hypertension. Risk Factors. Atherosclerosis.

\section{REFERÊNCIAS BIBLIOGRAFICAS}

1 - KAPLAN NM. Systemic hipertension: Mechanisms and diagnosis In BRAUNWALD E. Heart disease, 4th ed., WB Saunders, Philadelphia, p. 817-851, 1992.

2 - WILSON PWF et al. Twelve-year incidence of coronary heart disease in middle-aged adults during the era of hypertensive therapy: The Framingham Offspring Study. Am J Med 90: 11-16, 1991.

3 - KANNEL WB \& ABBOTT RD. Incidence and prognosis of unrecognized myocardial infarction. An update on the Framingham Study. N Eng J Med 311: 1144-1147, 1984

4 - KANNEL WB; DANNENBERG AL \& ABBOTT RD. Unrecognized myocardial infarction in hypertension: The Framingham Study. Am Heart J 109: 581-585, 1985.

5 - KANNEL WB; GORDON T \& SCHWARTZ MJ. Systolic versus diastolic blood pressure and risk of coronary heart disease: The Framingham Study. Am J Cardiol 27: 335-345, 1971.

6 - KANNEL WB et al. Systolic blood pressure, arterial rigidity and stroke. The Framingham Study. JAMA 245: 1225-1228, 1981.

7 - ZEHENDER $M$ et al. Ventricular tachyarrythymias, myocardial ischemia, and sudden cardiac death in patients with hypertensive heart disease. Clin Cardiol 18: 377-383, 1995.
8 - MESSERLI FH. Left ventricular hypertrophy, arterial hypertension and sudden death. J Hypertens 8 (suppl 7): S181-185; 1990

9 - MESSERLI FH et al. Hypertensionand sudden cardiac death: Increased ventricular ectopy activityin left ventricular hypertrophy. Am J Med 77: 18-22, 1984.

10 - McLenachan JM et al. Ventricular arrhythmias in patients with hypertensive left ventricle hypertrophy. N Engl J Med 317: 787-792, 1987.

11 - ZEHENDER $M$ et al. Prevalence of circadian variations and spontaneous variability of cardiac disorders and ECG changes suggestive of myocardial ischemia in systemic arterial hypertension. Circulation 85: 1808-1815, 1992

12 - JAMES MA \& JONES JV. Ventricular arrythmias in unteratednewly presenting hypertensive patients compared with a matched normal population. J Hypertens 7: 409-415, 1989.

13 - LE HEUZEY JH \& GUIZE L. Cardiac prognosis in Hypertyensive patients: incidence of sudden death and ventricular arrythmias. Am J Med 84 (suppl 1B): 65-68, 1988.

14 - SZLACHCIC $\mathrm{J}$ et al. Coronary morbidity and mortality,preexisting silent coronary artery disease, and mild hypertension. Ann Intern Med 110: 1017-1026, 1989.

Recebido para publicação em 16/01/96

Aprovado para publicação em 25/04/96 